\title{
Can Mindsponge approach benefit parapsychological research?
}

\author{
Tam-Tri Le \\ Centre for Interdisciplinary Social Research \\ Phenikaa University \\ Yen Nghia, Ha Dong District, Hanoi 100803, Vietnam
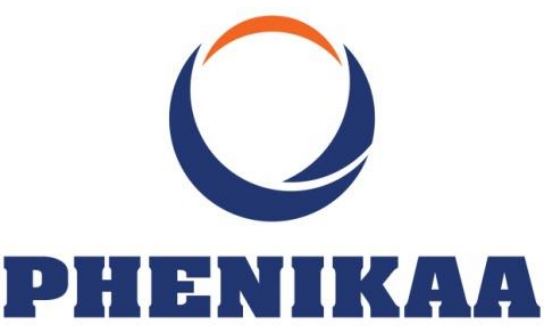 \\ UNIVERSITY
}

Written in Ho Chi Minh City, on May 24, 2021

OSF Preprints, DOI: 10.31219/osf.io/2dzsr

Parapsychology is often considered to be at the borderline of scientific inquiry. While most of the parapsychological researchers agree that certain paranormal phenomena do occur, the explanations have been "unsatisfactory" [1]. While conventional methodologies have difficulties in approaching this frontier, it leaves much room for innovation. Attempts at creating methodologies for quantifying parapsychological properties have also been growing, for example recently researchers at the Institute of Noetic Sciences created a scale for evaluating paranormal belief and experience [2]. Regarding theoretical framework, in particular the proposed mechanisms underlying such phenomena are currently lacking consensus, or not regarded as effectively applicable.

The Mindsponge theory $[3,4]$ is a framework of the mind's information processing mechanism. More detailed about its properties and application methods are presented in this paper investigating the psychological mechanism of suicidal ideation [5]. The Mindsponge framework is flexible enough to be potentially applicable even on abnormal psychological processes, as long as they involve some forms of cognitive shifting. It can also be applied on both micro levels (individual or small groups) and macro levels (society or culture).

Considering the special nature and constraints of parapsychological research, not all dimensions in the framework can be investigated (as with the current technology and understanding level). For example, the sources of information may need to stay unidentified as the environment is unknown. Furthermore, the multi-filtering apparatus in Mindsponge processes are already too 
complex to be directly examined or make clear distinctions in the cases of normal psychological phenomena, let alone paranormal cases. If applicable, it may be necessary to make modifications to the models in each study; but again, the high universality of the Mindsponge framework allows it to adapt to new perspectives which are likely to emerge when attempting to apply it on unfamiliar processes. This can become particularly beneficial considering the diversity and instability of parapsychological research's targets.

Another important point in parapsychology is the issues of subjectivity in general and the experimenter effect involving researchers. It is crucial in any scientific investigation to ensure an acceptable degree of objectivity, and parapsychological researchers often struggle with the fact that qualia cannot be accurately presented or measured by conventional methods. For this aspect, the Mindsponge approach can be advantageous by aligning the simulated information processing models in the researchers' minds during research designing with the measured values from those being studied. It may be even more effective if the researchers themselves are, as the same time, the studied targets (e.g. self-experimentation regardless of psi-related abilities).

\section{References}

1. Cardeña, E., Palmer, J., \& Marcusson-Clavertz, D. (Eds.). (2015). Parapsychology: A handbook for the 21 st century. McFarland \& Company, Inc., Publishers.

2. Wahbeh, H., Yount, G., Vieten, C., Radin, D., \& Delorme, A. (2020). Measuring extraordinary experiences and beliefs: A validation and reliability study. F1000Research, 8, 1741. https://doi.org/10.12688/f1000research.20409.3

3. Vuong, Q. H. (2016). Global Mindset as the Integration of Emerging Socio-Cultural Values Through Mindsponge Processes: A Transition Economy Perspective. In J. Kuada (Ed.), Global Mindsets: Exploration and Perspectives (pp. 109-126). Routledge. https://doi.org/10.4324/9781315736396-8

4. Vuong, Q. H., \& Napier, N. K. (2015). Acculturation and global mindsponge: An emerging market perspective. International Journal of Intercultural Relations, 49, 354367. https://doi.org/10.1016/j.ijintrel.2015.06.003

5. Nguyen, M.-H., Le, T.-T., Nguyen, H.-K. T., Ho, M.-T., Nguyen, H. T. T., \& Vuong, Q.-H. (2021). Alice in Suicideland: Exploring the Suicidal Ideation Mechanism through the Sense of Connectedness and Help-Seeking Behaviors. International Journal of Environmental Research and Public Health, 18(7), 3681.

https://doi.org/10.3390/ijerph18073681 